\title{
LOS DOCENTES COMO DISEÑADORES DE ENSEÑANZA
}

Laura Toro

Profesora de enseñanza primaria, Argentina

"El propósito de los grupos de aprendizaje cooperativo es fortalecer a cada integrante de manera individual. Los alumnos aprenden juntos para poder luego desempeñarse como individuos"

Johnson, $D$ 


\section{EL TRABAJO EN EQUIPO. LAS CONSIGNAS DE TRABAJO. LAS CLASES COOPERATIVAS}

Proyecto:"Somos periodistas"

Grado: $3^{\circ}$ año de EGB.

Año de implementación: 2002

Tiempo del proyecto: medio período del año escolar, dedicadas dos horas semanales al trabajo del mismo,

teniendo en cuenta flexibilidades organizativas.

\section{DIAGNÓSTICO DE SITUACIÓN}

La escuela donde se debía trabajar con este grado, tiene las características propias de la zona de pobreza y exclusión donde está inserta: pocos recursos económicos, código lingüístico de los alumnos restringido (lo cual hacía difícil la comunicación oral y escrita). Habían trabajado $1^{\circ}$ y $2^{\circ}$ año en grupo, pero no en trabajo cooperativo (los grupos no fueron designados por la docente, lo cual dio por resultado "grupos homogéneos en cuanto a características individuales").

No tenían acceso a libros de lectura y tampoco en las familias se leía el diario.

"El diario es utilizado como material de enseñanza que otorga márgenes de libertad al docente, divulga información, se ajusta al contexto educativo, integra contenidos y forma parte de un proyecto de trabajo".

"...la diferencia de materiales curriculares de otros que no son elaborados con fines pedagógicos, pero que, sin embargo, los docentes pueden integrarlos a sus prácticas de enseñanza para el desarrollo del currículum". ${ }^{2}$

Los alumnos estaban muy motivados a charlar en clase sobre noticias escuchadas en la televisión, en un país convulsionado.

Trabajaron un tiempo con computadoras donadas, donde escribían en Word pequeños textos, luego fueron robadas, pero se llegó a realizar varios trabajos con ellas.

Ante esta situación se decide implementar la utilización del diario como medio que actúe como facilitador del aprendizaje:

"No es el medio utilizado el elemento innovador, innovadora es la propuesta pedagógica en la que ese medio se inserta". 3

Se plantearon los siguientes objetivos:

Que el alumno logre:

- Reconocer las distintas secciones de un diario.

- Conocer el trabajo que realiza un periodista.

- Preparar distintos textos.

- Enriquecer el vocabulario.

- Participar en actividades grupales cooperativas en grupos heterogéneos.

- Investigar sobre diferentes temas, buscando y recopilando datos.

\footnotetext{
${ }^{1}$ ROTTEMBERG-ANIJOVICH. (2003)“Carpeta de trabajo UVQ”. Pág. 74

${ }^{2}$ AREA MOREIRA. (1999) "Los materiales curriculares en los procesos de diseminación y desarrollo del currículum”. Madrid. Ed. Síntesis. 
- Afianzar la lecto-escritura.

- Análisis de noticias.

- Trabajar todas las áreas de conocimiento, ínterdisciplinariamente.

- Realizar entrevistas a vecinos del barrio.

- Cotejar información de diarios con los otros medios de comunicación masiva: TV, radio, revistas.

- Estructurar las interacciones entre los alumnos, mediante equipos de trabajo.

- Lograr que los alumnos dependan unos de otros para lograr sus objetivos.

- Confeccionar un diario como trabajo final donde cada "grupo", en trabajo cooperativo, aportará el trabajo realizado sobre una determinada sección del diario que haya sido seleccionada.

\section{FORMACIÓN DE GRUPOS}

La escuela trabaja con un proyecto de "integración transdiciplinario" con otra escuela especial de la zona. Al trabajar con alumnos con "retardo mental leve", se trató de organizar cada grupo de acuerdo a distintas características y habilidades individuales de cada niño. La escuela trabaja con intenciones educativas de enseñar en la "diversidad".

"Los valores que fundamentan la educación en la diversidad son ante todo de carácter social y cultural (...) se parte de considerar como una riqueza, o cuanto menos como una característica de la realidad humana la diversidad de pensamientos, creencias, capacidades, procesos, intereses, etc. (...) se considera a la educación como un instrumento de promoción y desarrollo personal y social, y no como un instrumento de clasificación y jerarquización. "*

Se tuvo en cuenta una de las principales tareas que tiene el docente, que es la de integrar a los alumnos en grupos de aprendizaje, diagnosticar en qué punto de la curva de rendimiento se encuentra cada alumno, fortalecer los elementos básicos de la cooperación y hacer formar grupos heterogéneos: de acuerdo a su rendimiento escolar, a las diversas inteligencias múltiples sostenidas por Gardner.

"Una inteligencia implica la habilidad necesaria para resolver problemas o para elaborar productos que son de importancia en un contexto cultural o en una comunidad determinada.

En esta clase se forman "grupos de base cooperativo": el docente conociendo la "diversidad" de sus alumnos los agrupa. El funcionamiento de estos es a largo plazo y sus tipos de aprendizajes individuales heterogéneos. Los miembros son permanentes por el tiempo que demandará el proyecto. Esto permite que los alumnos establezcan relaciones responsables y duraderas que los motivarán a esforzarse en sus tareas y a progresar en el cumplimiento de sus obligaciones escolares.

Cada grupo eligió un nombre y hubo en el salón una tabla donde anotaron las secciones del diario elegidas con la cual trabajaron, para que no hubiese repeticiones.

Se buscará que los alumnos logren en este trabajo:

- Interdependencia positiva entre los alumnos.

- Responsabilidad individual y grupal.

\footnotetext{
${ }^{3}$ ROTTEMBERGANIJOVICH. (2003) "Carpeta de trabajo UVQ". Pág. 74

${ }^{4}$ MUÑOZ y MARUNY. (1993) "Cuadernos de pedagogía $n{ }^{\circ} 203$.
} 
- Interacción estimuladora entre compañeros y docente.

- Lograr prácticas interpersonales y grupales imprescindibles: cómo ejercer dirección, toma de decisiones, crear clima de confianza, comunicarse, manejar conflictos, sentirse motivados al hacerlo.

Ellos saben que en el plazo dado deberán presentar cada grupo su trabajo para armar el "periódico del grado".

\section{ORGANIZACIÓN ÁULICA}

Las mesas estaban distribuidas de manera que se favorezca el intercambio oral y el enriquecimiento grupal. Podíian pararse, hablar durante las actividades, mientras no molestasen a los demás miembros del salón. Los alumnos que trabajaron con las computadoras lo hicieron en forma organizada, por turnos, en el salón de Computación.

La cooperación es algo más que un método de enseñanza. Es un cambio básico en la "estructura organizativa" que afecta a todos los aspectos de la vida en el aula y en la institución toda.

\section{TRABAJO DE LOS ALUMNOS}

Los alumnos se repartieron y dividieron los trabajos de acuerdo a los temas que ellos dominaban, que habían elegido de acuerdo a los intereses individuales. Cambiaron opiniones y se organizaron según distintos roles antes de comenzar a realizar la actividades. Unos realizarían dibujos, otros escritura y recolección de datos y cada uno aportó los conocimientos previos para la realización del trabajo final.

No se observaron conflictos ya que los grupos permanecieron estables por un tiempo. Se conocían bien y sabían de virtudes y dificultades de cada uno de sus miembros. Se ayudaban mutuamente.

\section{ROL DEL DOCENTE DURANTE EL TRABAJO COOPERATIVO}

Observar continuamente el avance en la realización de los trabajos. Estar atento ante consultas requeridas, seguir de cerca el procedimiento. Sólo intervenir cuando sea necesario ante errores y para estimular el trabajo. Será mediador en conflictos que puedan surgir. Facilitar los diarios y otros elementos necesarios para el trabajo.

\section{OBSERVACIONES}

Se consiguió una visita a un periódico en forma gratuita. Los grupos solo debían organizarse cooperativamente para pagarse el transporte y así realizar la excursión.

Fue asombroso ver su organización para vender tortas, facturas, tortas fritas y una rifa de una canasta familiar con la que consiguieron los fondos para financiarse el viaje. Se comenzaba a observar el

\footnotetext{
${ }^{5}$ GARDNER, H. (1996) “Inteligencias múltiples. La teoría en la práctica”. Paidós. Barcelona.
} 
trabajo cooperativo de los grupos en otras actividades escolares. La unión y aporte de "cada uno" logró el objetivo deseado por "todos".

Realizaron la visita al Diario y quedaron sumamente motivados por todo lo observado y vivido en esa experiencia.

\section{EVALUACIÓN}

Los trabajos finales presentados me causaron emoción como docente por ver producciones tan enriquecedoras para mis alumnos. Al comenzar el proyecto, el $85 \%$ desconocía la organización de un diario y el contenido de las distintas secciones.

Cada grupo presentó varios trabajos de distintas secciones que les gustó trabajar. Se hizo una puesta en común y de a poco se fueron seleccionando por votación los trabajos que formarían el periódico final $^{6}$. También se encontraban mapas meteorológicos, clasificados, cine, reportaje a famosos, etc.

Se realizó durante la evaluación una charla abierta de cada "grupo" con sus vivencias, observaciones, inquietudes, problemas durante la realización. Así nos enriquecimos mutuamente para mejorar e innovar en otro proyecto o en el mismo. Se dio lugar al proceso de autoevaluación por parte del alumno, que aprenda a analizar sus trabajos y aprender de su propio proceso de reflexión. Se votaron los tres mejores trabajos que llevaron un premio y se destacó a los grupos que trabajaron más cooperativamente.

Si hablamos de heterogeneidad en la composición de un grupo escolar, lo debemos tener también en cuenta a la hora de evaluar: la gran variedad de estilos de aprendizaje, intereses, períodos de concentración, memoria, ritmos, tipos de inteligencia, niveles de conocimiento, etc.

\footnotetext{
${ }^{6}$ Se adjuntan algunos de los tantos trabajos que realizaron y fueron seleccionados, para las siguientes secciones: política, propagandas, historietas literarias (algunos hicieron chistes), deportes, mensajes, horóscopo.
} 

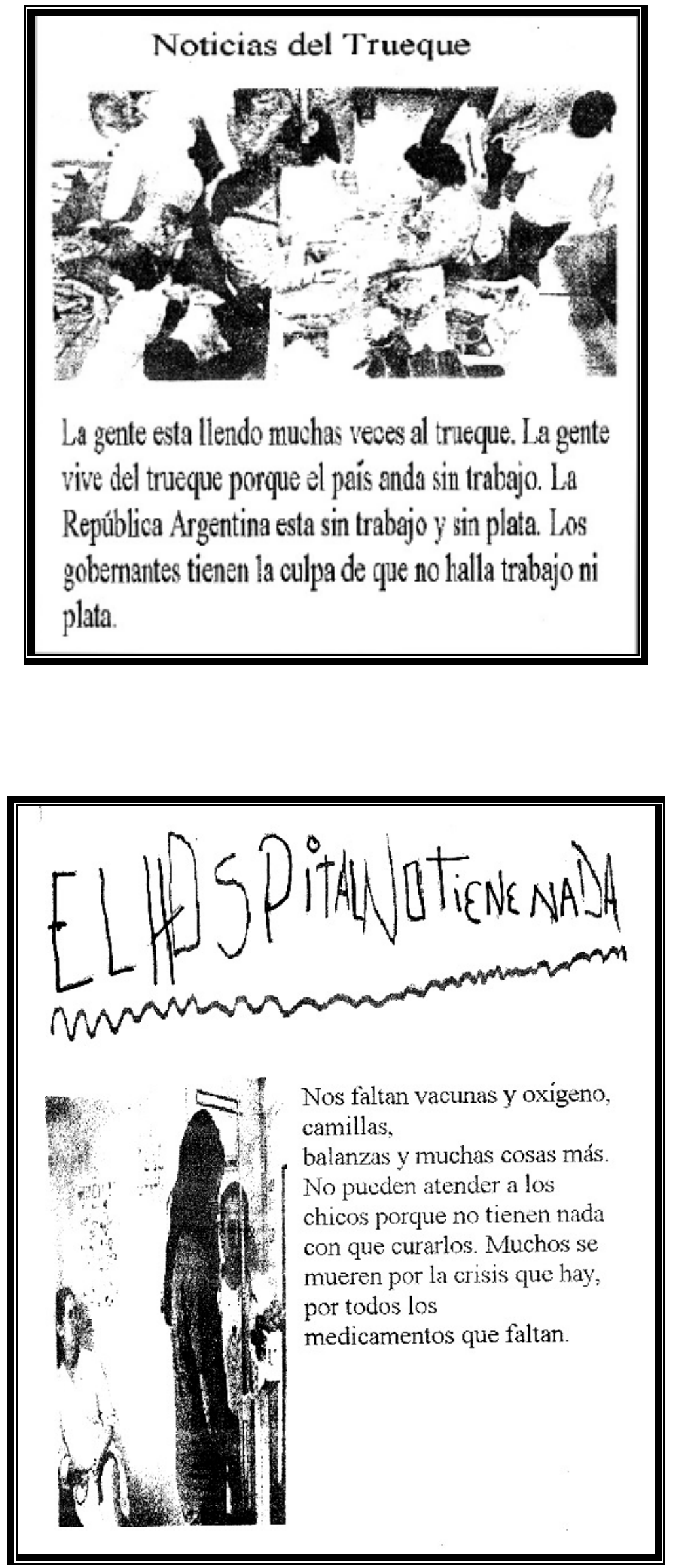


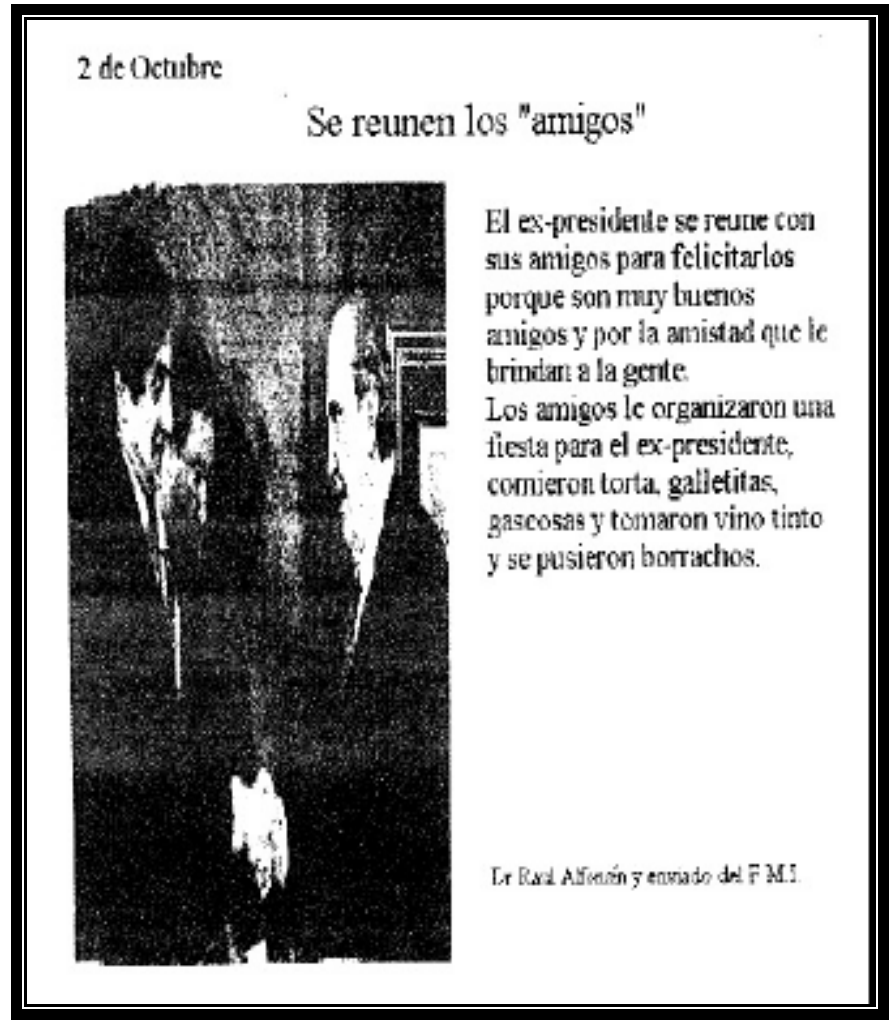

\section{Sucer mincada Coto do moNt 4326}

$\left[\begin{array}{l}40 \\ {[2}\end{array}\right] \quad W W, \operatorname{COM}, A R$
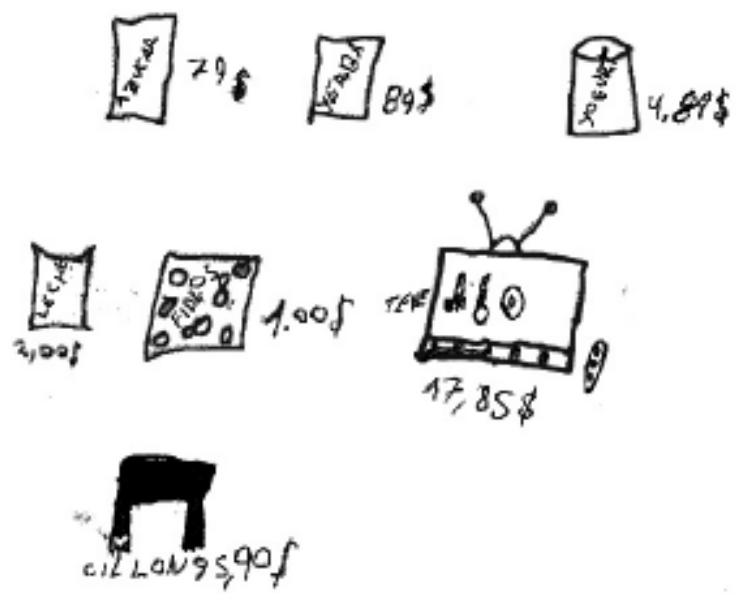

Compre ex coto el mifor sufermercado 

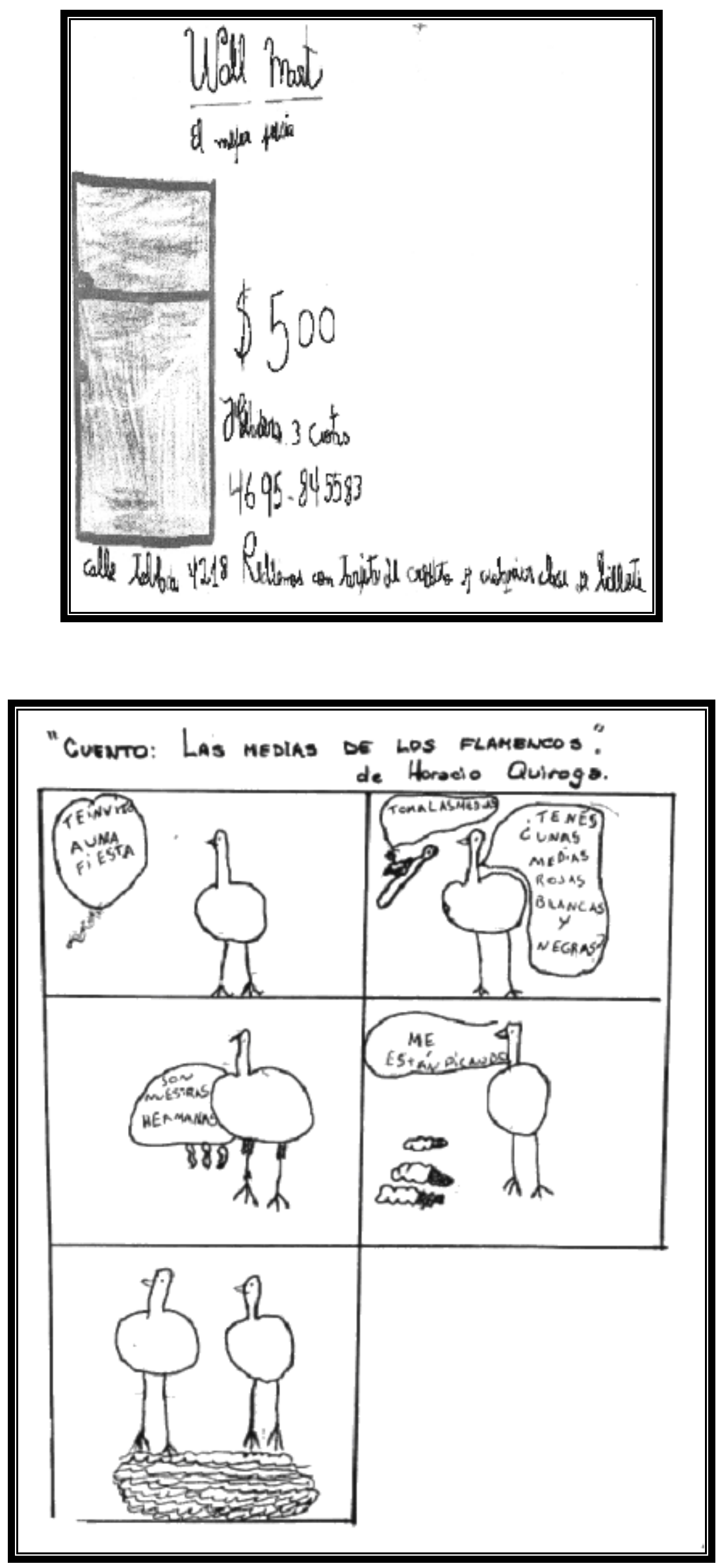


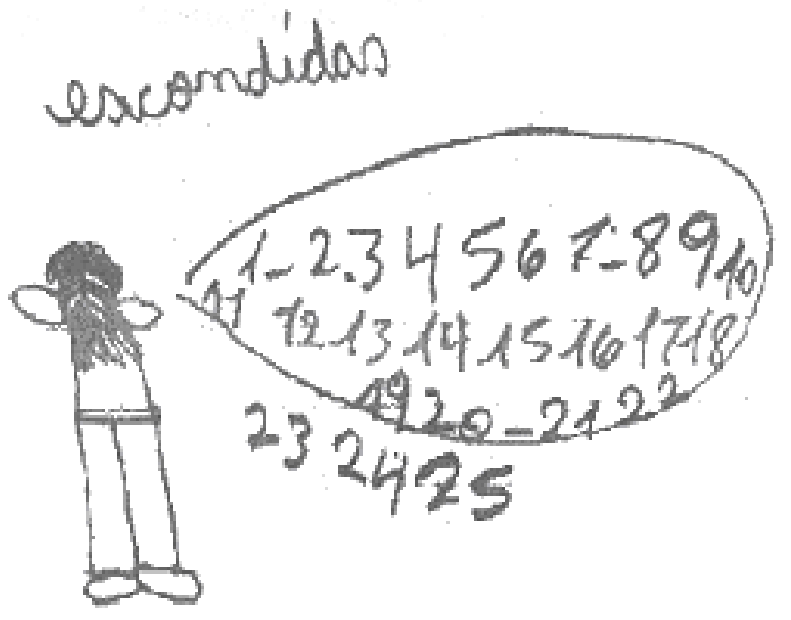

Las reglas son cuando los chicos juegan a

la escondida uno la cuenta por ejemplo hasta 25 o hasta cualquier otro número y después los que se escondieron mientras mira a otro lado el que cuenta tienen que tratar de librar para todos mi compañeros.

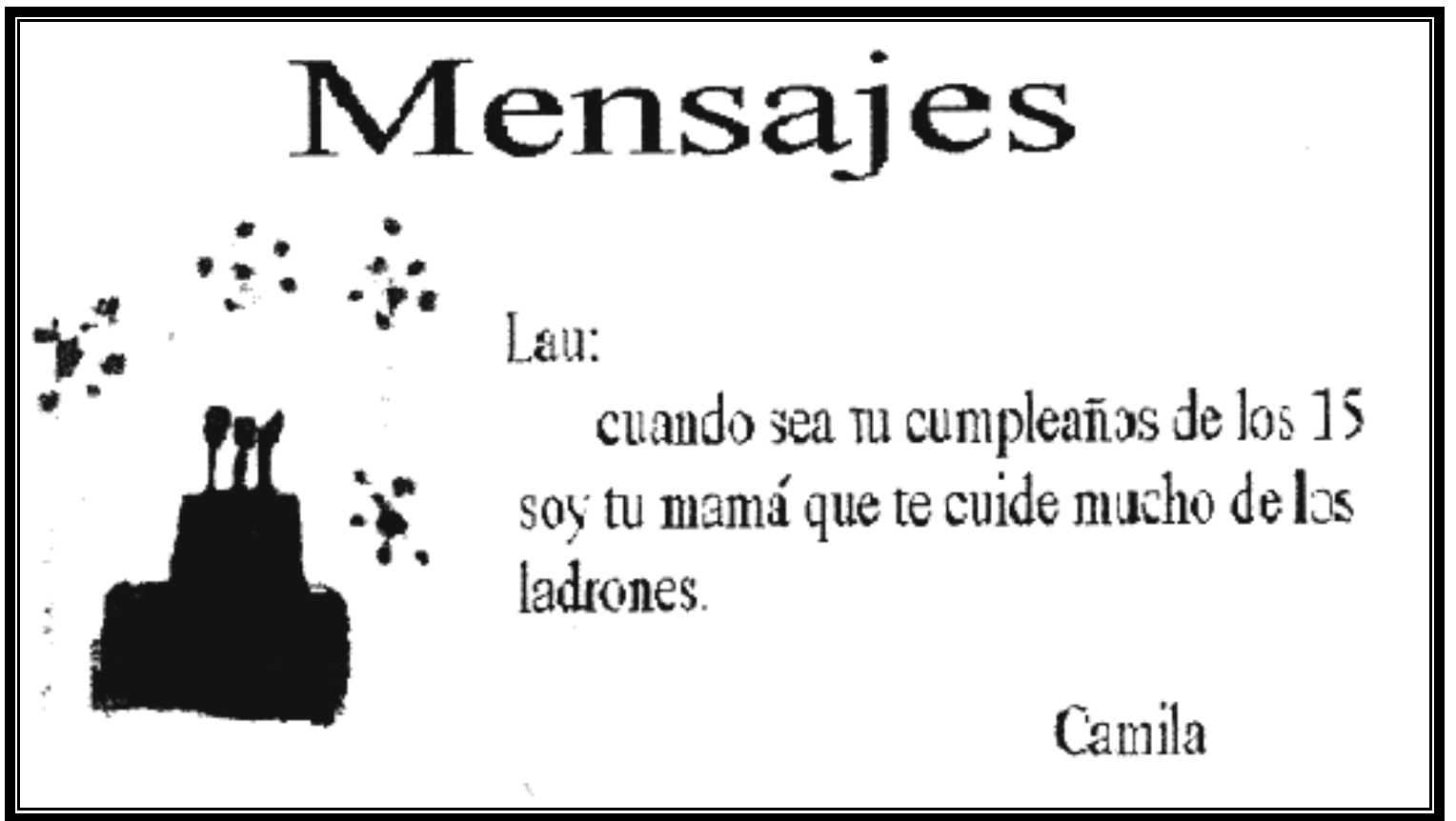




\section{Mensajes de Amor}

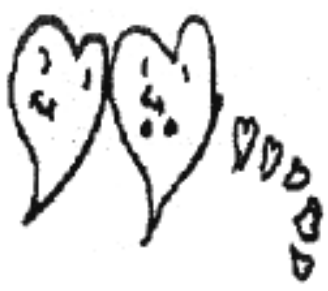

Querida mía:

te mando este mensaje

de amor para decirte

que te amo y te quiero

con todo el corazón.

$\mathrm{T}$ a Amo

Adrian

\section{HORÓSCOPO \\ Escorpio}

Que le vaya bien en cada día, que vaya a los hospitales, que le vaya bien en la casa, que encuentre alguien con quien confiar.

Estudio: que te defiendas en la escuela.

Trabajo: que te paguen bien y que no te roben.

Salud: que te respete la gente grande.

Amor: que si té cansas busques una buena tipa.

Suerte: que ganes plata, que tengas una linda familia.

\section{Capricornio}

Cuidado cuando comen que te puede doler la panza.

Mejora en el trabajo.

En el estudio te va a ir muy mal.

En el amor le va muy, pero muy, mal.

A mí me da suerte en el trabajo.

\section{Piscis}

Hoy tendrá un buen día en el trabajo, y se va a enamorar de una chica especial pero la chica no lo aceptó para nada.

Cómo te va a ir en la salud?, te va a ir muy bien, pero nada más vas a tener que estar a dieta.

Leo (24 de julio al 23 de agosto)

Usted debe cuidarse mucho. Haga ejercicios.

En amor usted tiene un chico que va a rondar por su cabeza.

Trabajo, dinero: usted tiene que jugar a la quiniela y asi ganará, y va a tener trabajo.

Hogar. sorpresa: usted va a tener la visita de una prima que nunca vino. 


\section{“TRABAJO Y APRENDIZAJE COOPERATIVO”}

Aprendizaje cooperativo: es una estrategia de gestión del aula que privilegia la organización del alumnado en grupos heterogéneos para la realización de las tareas y actividades de aprendizaje en el aula. Comparte la idea de agrupar a los alumnos en grupos heterogéneos en función del género y del ritmo de aprendizaje.

"Cuando éramos estudiantes, pedir ayuda a un compañero o tratar de ayudarse solía considerarse trampa y era algo que por entonces se penalizaba duramente(...) los docentes han comprobado que, para poder trabajar con éxito en grupos, los estudiantes necesitan que se les enseñen explícitamente las aptitudes para la cooperación, la colaboración y la resolución de conflictos, y también necesitan practicarlas constantemente antes de internalizarlas y convertirlas en comportamientos grupales de rutina..."

Johnson y sus colaboradores (1981) realizaron un interesante trabajo de revisión y llegaron a las siguientes conclusiones: ${ }^{8}$

- La cooperación es superior a la competición a la hora de fomentar el rendimiento y la productividad en todas las áreas, edades y niveles educativos.

- La cooperación es superior al aprendizaje individualista a la hora de promover logros y productividad cuando no es una tarea rutinaria de descifrar y corregir o cuando no se requiere una división del trabajo.

- La cooperación sin competición intergrupal parece promover un mayor logro y productividad que la cooperación con competición intergrupal, aunque es todavía escasa la investigación existente en este campo.

- No existe diferencia significativa entre las estructuras de metas interpersonales y las individualistas sobre rendimiento y productividad.

Dentro del clima del aula se observan situaciones cooperativas de aprendizaje, el liderazgo democrático, basado en la colaboración y en la decisión grupal de los alumnos, tiende a producir mejores decisiones, hacer que los miembros del grupo se sientan más obligados a cumplirlas, fortalecer la cohesión grupal y fomentar la motivación intrínseca, que los grupos dirigidos por lideres autoritarios (y centrados, por tanto en el profesor). Una característica central de los grupos de aprendizaje cooperativo es justamente el ejercicio de un liderazgo democrático, tanto por parte del profesor como por parte de los estudiantes dentro del grupo pequeño.

Johnson distingue cuatro tipos de grupos con los que gradualmente se llega al trabajo cooperativo de alto rendimiento. Estos grupos son:

- Grupo de pseudoaprendizaje: los alumnos acatan la directiva dada por el docente pero no tienen interés en hacerlo. Prima la competencia entre sí, cada alumno ve en los demás un competidor.

- Grupo de aprendizaje tradicional: se les solicita a los alumnos que trabajen en conjunto pero las tareas que se les asignan están estructuradas de tal modo que no requieren un verdadero trabajo conjunto.

- Grupo de aprendizaje cooperativo: el docente indica a los alumnos que trabajen juntos y ellos lo hacen voluntariamente. Saben que su rendimiento depende del esfuerzo de "todos" los miembros del grupo.

- Grupo de aprendizaje de alto rendimiento: cumple con todos los requisitos del grupo cooperativo pero, además obtiene rendimientos que superan las expectativas. Alto compromiso de los miembros entre sí y con el éxito del grupo.

\footnotetext{
${ }^{7}$ SHULMAN-LOTAN-WHITCOMB. (1999) "El trabajo en grupo y la diversidad en el aula". Bs. As.

${ }^{8}$ JOHNSON, D. "El aprendizaje cooperativo en el aula". Buenos Aires, Paidós
} 
Johnson (1980) señala que este tipo de aprendizaje fomenta en muy alto grado la interacción, la comunicación, la discusión entre los miembros del grupo: cuando se da a los alumnos la oportunidad para hacer planes conjuntamente, cuando se los estimula a discutir sus problemas y cuando se utilizan técnicas para fomentar la comunicación oral, la unidad del grupo se acrecienta.

Se ha demostrado empíricamente que el aprendizaje cooperativo proporciona más atracción interpersonal entre los estudiantes, incluso cuando los individuos se desagradan mutuamente al principio, las experiencias cooperativas fomentan más el agrado mutuo que las experiencias individualistas. Es mucho más frecuente el apoyo de los compañeros, y que este apoyo es importante para la implicación en la tarea y para la motivación de los estudiantes menos adelantados.

En síntesis, como afirma Johnson (1987) en las situaciones escolares, las ventajas más destacadas de las técnicas de aprendizaje cooperativo son:

- Aprendizaje de actitudes, valores, habilidades e información que no obtienen de los adultos.

- Oportunidades para practicar la conducta prosocial (ayudar, compartir, cuidar, etc. a los otros).

- Apreciación de situaciones y problemas desde otras perspectivas que las suyas propias.

- Pérdida progresiva del egocentrismo.

- Desarrollo de la autonomía y la capacidad de enfrentarse a las presiones del grupo.

- Desarrollo de actitudes positivas hacia los compañeros.

- Posibilidad de una mayor interdependencia y comunicación entre sus miembros.

- Prevención de alteraciones psicológicas y desajustes en el comportamiento cívico-social.

- Posibilidad de una más justa distribución del poder de la información, no centralizada en el profesor.

Es conveniente diferenciar estas técnicas de otras con las que usualmente suelen confundirse:

\begin{tabular}{|c|c|}
\hline Técnicas de aprendizaje cooperativo. & $\begin{array}{l}\text { Técnicas tradicionales } \\
\text { de aprendizaje grupal }\end{array}$ \\
\hline $\begin{array}{l}\text { - Interdependencia positiva: interés por el rendimiento } \\
\text { de todos los miembros del grupo. } \\
\text { - } \quad \text { Responsabilidad individual de la tarea asumida. } \\
\text { - } \text { Grupos heterogéneos. } \\
\text { - } \quad \text { Liderazgo compartido. } \\
\text { - } \quad \text { Responsabilidad de ayudar a los demás miembros del } \\
\text { grupo. } \\
\text { - Meta: aprendizaje del máximo posible. } \\
\text { - Enseñanza de habilidades sociales. } \\
\text { - Papel del profesor: intervención directa y supervisión } \\
\text { del trabajo en equipo. } \\
\text { - El trabajo se realiza en el aula. }\end{array}$ & $\begin{array}{l}\text { - Interés por el resultado del trabajo. } \\
\text { - Responsabilidad sólo grupal. } \\
\text { - Grupos homogéneos. } \\
\text { - Un solo líder. } \\
\text { - Elección libre de ayudar a los compañeros. } \\
\text { - Meta: completar la tarea asignada. } \\
\text { - Se da por supuesto que los sujetos poseen } \\
\text { habilidades interpersonales. } \\
\text { - Papel del profesor: evaluación del producto. } \\
\text { - El trabajo se realiza fuera del aula. }\end{array}$ \\
\hline
\end{tabular}




\section{CONCLUSIONES}

En el curso donde fue experimentado este tipo de aprendizaje, el clima del aula mejoró sensiblemente, puesto que los alumnos incrementaron positivamente su autoestima y los lazos de unión con sus compañeros del aula, reduciéndose de modo significativo las hostilidades, tensiones y prejuicios raciales, tan presentes en la actualidad, sobre todo en las clases de alumnos adolescentes.

"Los estudiantes que aprenden en grupos también demuestran tener habilidades sociales y una mayor aceptación interracial e interétnica".9

Este método incrementa la atracción de los estudiantes hacia los compañeros y hacia la escuela, mejora su rendimiento académico, disminuye su competitividad y les ayuda a ver a sus compañeros como fuentes de aprendizaje. Fomentó también la unión de las familias de los chicos que formaban los "grupos cooperativos" ya que ellas participaron activamente en el proyecto, como en la realización de la excursión. Se logró formar "grupos de aprendizaje cooperativo de alto rendimiento".

Los alumnos parecen mostrar una mayor capacidad para ponerse en el lugar de los demás (desarrollo moral) y tienden a hacer atribuciones de ensalzamiento del yo tanto por si mismos como para sus compañeros de clase.

Tres grandes beneficios observados en la implementación de este proyecto: estuvo asociado a la enseñanza para la comprensión, desarrollo del pensamiento, entender a la enseñanza como un proceso.

El alumno tuvo total protagonismo y un papel activo donde el docente perdió cierto grado "central", para ubicarse en el rol de orientador, guía, acompañante. Esto requiere humildad, escucha y capacidad de asombro, porque enseñar de esta manera hace posible que muchas veces los alumnos nos sorprendan y aprendamos de ellos.

El aprendizaje tendrá que ver con el compromiso, flexibilidad y crecimiento permanente tanto del docente como del alumno.

\section{BIBLIOGRAFÍA}

ANGUlo RASCO, José FÉliX Y BlANCO, NieVes (1994), “Cap. 12: Materiales curriculares: los libros de texto", en: Teoría y desarrollo del curriculum, Málaga, Aljibe, pp. 263 a 280.

CAMILLONI, A. Y OTRAS (1998), "La calidad de los programas de evaluación y de los instrumentos que los integran", en: La evaluación de los aprendizajes en el debate didáctico contemporáneo, Buenos Aires, Paidós

ROTTEMBERG-ANIJOVICH. “ Carpeta de trabajo”. Universidad Virtual Quilmes. Año 2003

DE KETELE, J. M. (1984), "Cap. 1: "Educar, evaluar, observar: el marco de la problemática” y "Cap. 2: Evaluar para educar: ¿por qué?, ¿qué?, ¿quién?, ¿cómo?", en: Observar para educar, Visor, Madrid, pp. 13 a 27 у pp. 29 a 32.

Del Carmen, L. (1996), "Cap. 2: El análisis, selección, secuenciación y organización de contenidos en el proceso de elaboración del currículo" y "Cap. 9: Propuesta de criterios para la secuenciación de

\footnotetext{
${ }^{9}$ SHULMAN-LOTAN-WHITCOMB. (1999)
} 
contenidos", en: El análisis y secuenciación de los contenidos educativos, ICE, Barcelona, pp. 23 y pp. 161 a 195.

Del Carmen, L. (1996), "Cap.5", en: El análisis y secuenciación de los contenidos educativos, Cuadernos de Educación, № 21, ICE, Barcelona

Fenstermacher G. Y SOltis J. (1999), “Cap.1: Enfoques de enseñanza”, “Cap.2: El enfoque del ejecutivo” y "Cap.3: El enfoque del terapeuta”, "Cap. 4: El enfoque liberador”, en Enfoques de enseñanza, Amorrortu, Bs. As., pp. 15 a 29, pp. 31 a 53 pp. 55 a 77, Pág. 79 a 105.

GARDNER H. (1993), "Capítulo 1" en: La mente no escolarizada, Barcelona, Paidós

GASKINS y ELLIOT (1999), “Cap. 4: Estrategias cognitivas y metacognitivas", "Cap.5: La puesta en práctica de la enseñanza de estrategias", "Cap. 11: Desarrollo de un currículo que articule contenidos y estrategias", en: Como enseñar estrategias cognitivas en la escuela, Paidós, Bs. Aires

MARTíneZ BONAFÉ, J. (1992) “¿Cómo analizar los materiales?” en Cuadernos de Pedagogía № 203.

Salomon, G., Perkins, D. Y Globerson, T. (1992), "Coparticipando en el conocimiento: la ampliación de la inteligencia humana con las tecnologías inteligentes", en: Comunicación, lenguaje y educación № 23

STONE WISKE, M. (1999), “Cap. 2: ¿Qué es la comprensión?”, “Cáp.3: ¿Qué es la Enseñanza para la Comprensión?”, “Cap. 4: ¿Cómo aprenden los docentes a enseñar para la comprensión?”, en: Martha Stone Wiske, Karen Hammerness, Daniel Gray Wilson, La enseñanza para la comprensión, Paidós, Buenos Aires, pp. 69, 95 y 127.

JOHNSON, D (1999), "Cap.1. El concepto de aprendizaje cooperativo" y "Cáp.9 La puesta en práctica de la clase cooperativa", en: El aprendizaje cooperativo en el aula, Buenos Aires, Paidós pp.13-30 y pp. 8998http: // www.clarin.com.ar

http: // www.lanacion.com.ar

http: // www.galeon. hispavista.com

AREA MOREIRA (1999) "Los materiales curriculares en los procesos de diseminación y desarrollo del currículum". En Escudero, J Diseño, desarrollo e innovación del currículum. Madrid. Ed. Síntesis.

SHULMAN-LOTAN-WHITCOMB (1999): "El trabajo en grupo y la diversidad en el aula". Buenos Aires. Amorrortu. 


\title{
Contactar
}

Revista lberoamericana de Educación

\author{
Principal OEI
}

